Archived version from NCDOCKS Institutional Repository http://libres.uncg.edu/ir/asu/

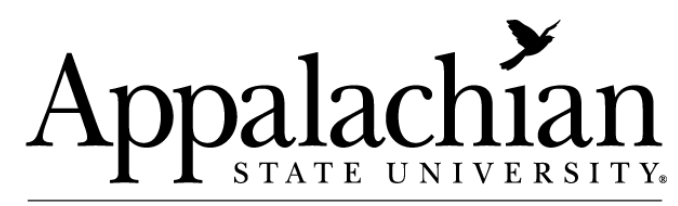

$\overline{B \text { O O N E, N O R T H C A O L I N A }}$

\title{
"Prologues To A Bad Voice": Effect Of Vocal Hygiene Knowledge And Training On Voice Quality Following Stage Performance
}

\author{
By: Balaji Rangarathnam, Towino Paramby, and Gary H. McCullough
}

\begin{abstract}
The purpose of this investigation was to determine the effects of intensive stage rehearsal and performance on perceptual, acoustic, and aerodynamic measures of voice, and to determine the impact of knowledge and practice of vocal hygiene on measures of voice during intensive vocal performance. Nineteen stage actors who were participating in the Arkansas Shakespeare Theatre festival took part in the study. Each participant completed auditory-perceptual, acoustic, aerodynamic, and quality of life measures before and after 1 month of intensive rehearsals and stage performances. They also completed a questionnaire documenting their vocal use, vocal hygiene, and previous vocal training, if any. Subjects demonstrated statistically significant deterioration in auditory-perceptual measures and mean expiratory airflow. Other acoustic measures trended toward poorer outcomes after the performances; however, these were not statistically significant. Knowledge of vocal hygiene and vocal training did not have an impact on the change in vocal measures. Conclusions: Stage performances do impact vocal outcomes with reduction in quality and efficient use of airflow for voice production. Knowledge and practice of vocal hygiene have some impact on these changes; however, vocal hygiene may not be the best preventive strategy of potential phonotrauma in this subject population.
\end{abstract}

Rangarathnam, B., Paramby, T., and McCullough, G. (2018). "Prologues to a Bad Voice": Effect of Vocal Hygiene Knowledge and Training on Voice Quality Following Stage Performance. Journal of Voice, Volume 32, Issue 3, May 2018, pages 300-306. https://doi.org/10.1016/i.jvoice.2017.05.026. Publisher version of record available at: http://www.sciencedirect.com/science/article/pii/S089219971730053X 


\title{
"Prologues to a Bad Voice": Effect of Vocal Hygiene Knowledge and Training on Voice Quality Following Stage Performance
}

\author{
*Balaji Rangarathnam, †Towino Paramby, and ¥Gary H. McCullough, *Greenville and $\ddagger$ Boone, North Carolina, and \\ $\dagger$ Conway, Arkansas
}

\begin{abstract}
Summary: Purpose. The purpose of this investigation was to determine the effects of intensive stage rehearsal and performance on perceptual, acoustic, and aerodynamic measures of voice, and to determine the impact of knowledge and practice of vocal hygiene on measures of voice during intensive vocal performance.

Methods. Nineteen stage actors who were participating in the Arkansas Shakespeare Theatre festival took part in the study. Each participant completed auditory-perceptual, acoustic, aerodynamic, and quality of life measures before and after 1 month of intensive rehearsals and stage performances. They also completed a questionnaire documenting their vocal use, vocal hygiene, and previous vocal training, if any.

Results. Subjects demonstrated statistically significant deterioration in auditory-perceptual measures and mean expiratory airflow. Other acoustic measures trended toward poorer outcomes after the performances; however, these were not statistically significant. Knowledge of vocal hygiene and vocal training did not have an impact on the change in vocal measures.

Conclusions. Stage performances do impact vocal outcomes with reduction in quality and efficient use of airflow for voice production. Knowledge and practice of vocal hygiene have some impact on these changes; however, vocal hygiene may not be the best preventive strategy of potential phonotrauma in this subject population.
\end{abstract}

Key Words: Stage actors-Phonotrauma-Vocal hygiene-Vocal training-Stage performances.

\section{INTRODUCTION}

"Prologues to a Bad Voice"1 seems an apt reference to the potential problems stage actors face maintaining their voices night after night. High vocal demands coupled with inappropriate use of voice or inadequate knowledge regarding vocal care can, potentially, lead to significant voice problems, poor performance, and eventually lack of work for professional stage actors. Changes in voice quality in professional voice users, especially singers, have been of significant interest to voice researchers for many years. ${ }^{2,3}$ Systematic investigations of various parameters of voicing before and after extended periods of vocally demanding performances, however, are sparse in the extant literature. The Arkansas Shakespeare Theatre brings professional actors from around the country to the campus of the University of Central Arkansas every spring for 6 weeks, during which three Shakespearean plays and one popular play or musical are performed. This provided the opportunity to measure voice quality and related measures over an intensive 6-week period in actors with a range of experience, as well as a range of knowledge in appropriate care of the professional voice.

Stage actors rely heavily on their voices not only to convey communicative information but also to entertain, engage, and enrapture audiences. Controlling and coordinating various speech behaviors including respiration, phonation, articulation, resonance,

From the *Department of Communication Sciences and Disorders, East Carolina University, Greenville, North Carolina; $†$ Department of Communication Sciences and Disorders, University of Central Arkansas, Conway, Arkansas; and the $\ddagger$ Beaver College of Health Sciences, Appalachian State University, Boone, North Carolina.

Address correspondence and reprint requests to Balaji Rangarathnam, Department of Communication Sciences and Disorders, East Carolina University, 600 Moye Blvd., Mailstop 668, Greenville, NC 27834. E-mail: rangarathnamb@ecu.edu and prosody ${ }^{4}$ to achieve these goals over an extended period of time can be detrimental to the healthy voice. Singing, dancing, emoting, crying, and laughing, sometimes at the same time, can lead to inappropriate and excessive muscular activity associated with voicing, which can further fatigue the vocal folds and render ongoing performances challenging and even detrimental to healthy vocal function. ${ }^{5}$ Stage actors exceed their intensity levels on theatrical reading tasks, and female participants often lower their pitch to inappropriate levels, whereas male actors raise their pitch to inappropriate levels. ${ }^{6,7}$ These changes result in vocal fatigue in actors, as well as poor physical and emotional health and performance. ${ }^{8}$

Most stage actors and other professional voice users traditionally acquire some training on vocal hygiene. Vocal hygiene education has traditionally been used as both preventive strategy for reducing future voice problems and a management strategy as part of comprehensive treatment approach for voice disorders. ${ }^{9}$ A vocal hygiene program typically includes discussions and directions regarding the amount and type of voice use; phonotraumatic behaviors - which may include throat clearing, loud and inappropriate voice use, cheering, and screaming; hydration; and lifestyle practices — such as reduction of caffeinated beverages, diet patterns, elimination of drug use and alcohol, among others. ${ }^{9,10}$ Several of these actors also undergo specific vocal training to "conserve" their voices. Vocal training can be broadly described as the use of maneuvers that aim at changing the existing voice production to prevent tissue damage and subsequent voice disorders and promote optimal voice production. ${ }^{11}$ Vocal training may encompass "warm-up" exercises, basics of vocal hygiene, efficient projection of voice, and resonant voice production, among others. ${ }^{11}$

A few investigations have studied the voices of stage actors and the impact of vocal hygiene and training. Roy and colleagues ${ }^{12}$ 
studied the effect of "vocally violent behaviors" and vocal hygiene training on the voices of 27 actors. Vocally violent behaviors were defined as grunting, groaning, sobbing, and shouting. Voice measures were obtained at four time points: pre-training or prevocal violence, pre-training or post-vocal violence, posttraining or pre-vocal violence, and post-training or post-vocal violence. The authors observed that the vocally violent behaviors did not impact the voices except for higher frequencies where an expanded fundamental frequency range was noted. Vocal hygiene training, however, yielded statistically significant reduction in frequency and amplitude perturbation and noise levels in the voice. The results suggest positive effects of vocal hygiene education in stage actors even though the impact of "vocal violence" was minimal.

Timmermans et $\mathrm{al}^{13}$ reported a profile of vocal characteristics from a relatively large sample of 86 occupational voice users in training. Of these 86 subjects, 24 subjects were stage actors in training and 12 subjects were musical actors in training. The data for these 36 subjects were reported under the category of elite vocal performers in training. The authors reported that eight of the elite vocal performers in training had either an organic or an inflammatory lesion in the larynx, a dysphonia severity index of 2.5 (males) and 2.2 (females), and a maximum phonation duration of 16.3 (males) and 13.9 (females). Although the results do not suggest extensive impairments in the voices, it should be noted that the subjects reported in this study were in training and were just beginning their vocal performances. These data could provide insights into the voices as a baseline measure before any changes happen as an effect of vocally demanding training or performances.

Laukkanen and colleagues ${ }^{11}$ studied 12 student actors and reported positive outcomes from vocal training. The participants were divided into two groups - one of the groups received behavioral vocal training focused on voice projection, use of a "ringing" tone in voice (use of resonant voice characteristics), and the use of an easy voice, whereas the second group received vocal training using biofeedback. Both the groups improved on sound energy, particularly at $3-5 \mathrm{kHz}$ frequency range, and "voice quality," as judged by two listeners on an 11-point scale. The results suggest that vocal training could help with a more optimal production of voice, which could potentially prevent the incidence of voice disorders.

Studies documenting actual changes in vocal measures are scant. That is, data relating to a comprehensive acoustic, perceptual, and aerodynamic assessment of stage actors before and after an extended period of vocally demanding performances are lacking in the literature. Such data could add to the current body of literature, providing new insights into vocal fatigue in actors and critical data on the role of vocal training and vocal hygiene. The purpose of this investigation was twofold: first, to determine the effects of intensive stage rehearsal and performance on perceptual, acoustic, and aerodynamic measures of voice; and second, to determine the impact of knowledge and practice of vocal hygiene by the actors on measures of voice during intensive vocal performance. We did not offer any training or vocal hygiene education as part of the study design; rather, we sought to investigate the impact of any vocal hygiene the actors were already employing and any vocal training they may have received before the study. We hypothesized that perceptual, acoustic, and aerodynamic measures of voice would be significantly worse after the intensive period of stage acting than at the beginning, and that participants with more vocal training and more education in vocal hygiene would exhibit significantly fewer negative changes in perceptual, acoustic, and aerodynamic measures than those without such training and education.

\section{Participants}

\section{METHOD}

Nineteen stage actors (nine males, nine females, and one subject withdrawn from the study) age 19-74 years were recruited from The Arkansas Shakespeare Theatre Company. All participants signed informed consent forms approved by the University of Central Arkansas Institutional Review Board. Participants' years of acting professionally varied from 1 year to greater than 10 . Thirteen subjects reported they received training on vocal hygiene at some level, and 16 subjects testified receiving voice training or coaching (Table 1). The following assessment measures were obtained before and after 1 month of intensive performances and rehearsals.

\section{Assessment measures}

To assess knowledge of vocal hygiene, subjects were asked to fill out a Vocal Hygiene and Training Questionnaire (Appendix) during their assessment. This questionnaire was designed for this study and consisted of various vocal hygiene principles and questions on vocal training.

\section{Auditory-perceptual assessment}

Auditory-perceptual assessment was completed using the Consensus Auditory-Perceptual Evaluation of Voice (CAPE-V). ${ }^{14}$

Voice samples included sustaining /a/ and /i/ for at least 3 seconds, reading six sentences, and conversing. All voice samples were digitally recorded onto the Sona-Speech program (KayPENTAX, Inc, Lincoln Park, NJ) in a quiet room with minimal ambient noise. The default calibration settings of Sona-Speech were used, and the microphone was kept at a consistent distance of approximately 6 inches from the speaker's mouth. CAPE-V measures included an overall rating of voice quality and ratings of perceptual roughness, strain, breathiness, and nasality. Recordings were coded without identifying information and were listened to and rated by an experienced speechlanguage pathologist (SLP) (author GHM). The SLP listened to each pair (pre and post) of recordings for each participant at the same time but was blinded to the time point of recording (pre $v s$. post). In other words, the rater had two samples to rate without the knowledge of whether the sample was obtained before or after the 6-week period. Only the overall severity measure was considered for analysis purposes. Inter-rater reliability was established by a second listener, a certified SLP with at least 5 years of experience in voice, who rated the recordings. The second rater rated about $33 \%$ of the samples; the samples were presented the same way as they were for the first rater. 
TABLE 1.

Demographic Information

\begin{tabular}{|c|c|c|c|c|c|c|c|}
\hline Subject \# & Age & Gender & $\begin{array}{l}\text { Years Acting } \\
\text { Professionally }\end{array}$ & $\begin{array}{l}\text { Weeks of } \\
\text { Performance } \\
\text { Each Year }\end{array}$ & $\begin{array}{l}\text { How Often Do } \\
\text { Performances } \\
\text { Involve Singing? }\end{array}$ & $\begin{array}{c}\text { Received } \\
\text { Training on } \\
\text { Vocal Hygiene? }\end{array}$ & $\begin{array}{c}\text { Received Voice } \\
\text { Training or } \\
\text { Coaching? }\end{array}$ \\
\hline 2 & 74 & M & $>10$ & $<10$ & Minimal & Yes & Yes-many years \\
\hline 3 & 60 & M & $>10$ & $>40$ & Half & Yes & Yes-38 y \\
\hline 4 & 37 & $\mathrm{~F}$ & $>10$ & $10-20$ & None & Yes & Yes-limited or periodic \\
\hline 7 & NR & NR & NR & NR & NR & NR & NR \\
\hline 8 & W & W & W & W & W & W & W \\
\hline 9 & 27 & $\mathrm{~F}$ & $3-4$ & $>40$ & Minimal & Yes & Yes-4 y \\
\hline 10 & 26 & $\mathrm{~F}$ & $5-10$ & $21-30$ & Half & Yes & Yes-10+y \\
\hline 11 & 46 & $\mathrm{~F}$ & $>10$ & $>40$ & Minimal & No & No \\
\hline 16 & 23 & $\mathrm{~F}$ & $1-2$ & $10-20$ & Most & No & Yes-4 y \\
\hline 17 & 50 & $F$ & $>10$ & $<10$ & Most & Yes & Yes-36 y \\
\hline 18 & 35 & $\mathrm{~F}$ & $5-10$ & $10-20$ & All & NR & Yes -9 y \\
\hline 19 & 39 & $\mathrm{M}$ & $1-2$ & $10-20$ & Minimal & No & Yes $-5 y$ \\
\hline
\end{tabular}

Abbreviations: NR, no response to questionnaire; W, withdrawn from study.

\section{Acoustic voice measurement}

The multidimensional voice profile and real-time pitch of the SonaSpeech program (KayPENTAX Inc) were used to collect acoustic measures. For the multidimensional voice profile analysis, the voice sample consisted of /a/ vowel sustained for 5 seconds, which was analyzed for noise-to-harmonic ratio and frequency or intensity perturbation measures. For the real-time pitch analysis, the samples consisted of reading the Rainbow passage and counting from 1 to 10. Habitual pitch and intensity, mean speaking frequency and intensity, and frequency or amplitude range were recorded. The default calibration settings of the Sona-Speech program were used, and the microphone was kept at a consistent distance of approximately 6 inches from the speaker's mouth.

\section{Aerodynamic measurement}

Aerodynamic assessment of phonatory airflow and laryngeal airway resistance was derived using the Phonatory Aerodynamic System Model 6600 (KayPENTAX, Inc). The following tasks were conducted:

(1) Maximum sustained phonation (MSP): Participants were instructed to take a deep breath, then to produce a sustained open vowel (/a/) at a comfortable pitch and loudness for as long as they could sustain voicing in one breath.

(2) Comfortable sustained phonation (CSP): Participants were instructed to take a deep breath, then to produce a sustained open vowel (/a/) at a comfortable pitch and loudness for at least 5 seconds once data capture was initiated.

(3) Voicing efficiency (VOEF): Participants were instructed to repeat the voiced vowel /a/ and the voiceless stop plosive /p/ nine times in vowel-consonant format (ie, /apapapapapapapa/), placing equal stress on each syllable, as described by Zraick and colleagues. ${ }^{15}$ To ensure consistent rhythm, participants were trained on the speaking task until they produced the syllable trains evenly and at a comfortable loudness level.

Three trials of each of the foregoing tasks were conducted, and the average of the three trials was used for analysis. Subjects were provided with instructions for each task before every trial. Mean phonatory airflow (L/s) was derived from CSP and MSP tasks individually. CSP protocol was based on analysis of a sustained portion of voicing that was comfortable in pitch and loudness for the subject. We were, however, interested in the total expiratory volume and phonation time, as well, and, therefore, the MSP protocol was also employed. Estimates of subglottal pressure, mean phonatory sound pressure level, laryngeal airway resistance, and phonatory airflow were derived from the VOEF task. Measures of average peak (intra-oral) air pressure during adjacent productions of the consonant /p/ (across syllables 2-8) provided an estimate of subglottal pressure. ${ }^{15}$ Mean airflow during voicing was derived from the oral airflow measures during the vowel segments. The airflow signal was examined to ensure a baseline (zero) was reached for each pressure peak, so as to not underestimate subglottic pressure.

\section{Log book}

In addition to the above assessments, all participants were asked to keep a logbook, which included all food and beverages consumed each day, medications, and voice use. Actors were asked 
to $\log$ the estimated length of rehearsals or performance and the type of voice use (singing $v s$. speaking).

\section{Statistical analysis}

To address specific aim 1 (to determine the effects of intensive stage rehearsal and performance on perceptual, acoustic, and aerodynamic measures of voice), we performed Wilcoxon signed rank test to compare pre-post variables on each of these outcome measures. We considered the maximum phonation duration, the overall severity of CAPE-V, noise-to-harmonic ratio, vocal turbulence index, jitter\%, shimmer\%, mean airflow rate obtained on the MSP task and the comfortable sustained phonation tasks, aerodynamic efficiency, aerodynamic power, and aerodynamic resistance obtained using the VOEF tasks for the purposes of the analysis. To address specific aim 2 (to determine the impact of knowledge and practice of vocal hygiene by the actors on measures of voice during intensive vocal performance), we divided the subjects into two groups depending on their knowledge of vocal hygiene and vocal training using responses on the vocal hygiene questionnaire. Specifically, we classified subjects on each question on the questionnaire into two groups as outlined in Table 1. We then administered nonparametric Kruskal-Wallis analyses to determine how the changes in vocal measures (described in the analyses for aim 1) were impacted by knowledge and practice of vocal hygiene and vocal training.

\section{RESULTS}

\section{Analysis for specific aim 1: comparison of pre-post variables on outcome measures}

The results of the Wilcoxon signed ranks test (Table 2) show statistically significant deterioration in the overall severity on CAPE-V $(Z=-2.431, P=0.015)$ and mean airflow on MSP $(\mathrm{Z}=-3.622, P<0.001)$. Inter-rater reliability of the perceptual analysis on CAPE-V was "excellent" based on intraclass correlation coefficients ( 0.77 for pre-performance CAPE-V severity and 0.81 for post-performance CAPE-V severity).

Maximum phonation duration was also substantially reduced in the post-treatment measurement $(\mathrm{Z}=-1.728, P=0.084)$, but not to the point of statistical significance. None of the other measures demonstrated statistically significant changes. Several measures, however, trended toward deterioration even though statistical significance was not met. These measures include harmonics-to-noise ratio (pre median score of 0.133 to post median score of 0.121 ), vocal turbulence index (pre median score of 0.03 to post median score of 0.035 ), and aerodynamic resistance (pre median score of 48.19 to post median score of 55.36).

\section{Analysis for specific aim 2: Kruskal-Wallis analysis to} determine the effect of vocal hygiene and training

The only measures that were significantly impacted as measured by Kruskal-Wallis tests were increased vocal turbulence in individuals with higher levels of alcohol consumption $(P=0.24)$ and reduced mean airflow on the MSP task in individuals with poor water intake $(P=0.38)$. None of the other measures were significantly impacted by the practice of vocal hygiene or vocal training.

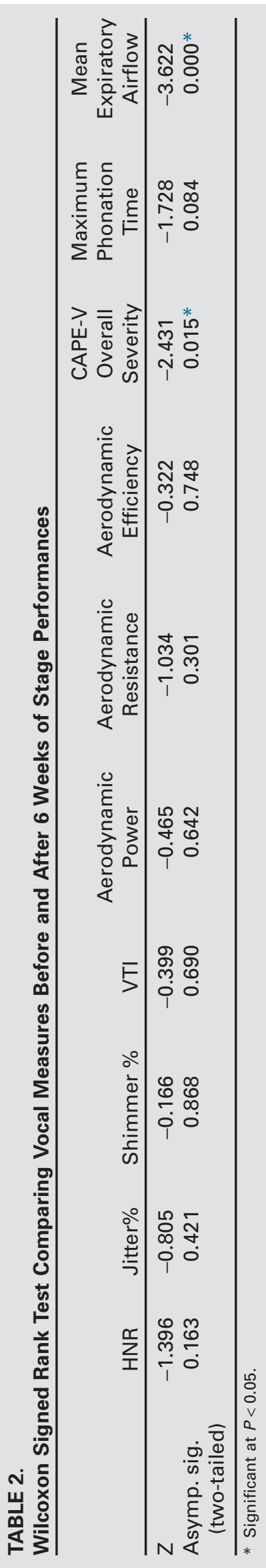




\section{DISCUSSION}

This study examined the effects of intensive vocal use during rehearsals in stage actors and the potential impact of hygienic vocal practices and training. Results indicate that auditoryperceptual and aerodynamic measures deteriorate after stage performances and rehearsals; however, these changes are present, for the most part, regardless of an individual's practice of vocal hygiene and vocal training.

Analysis for the first specific aim suggests that voices of stage actors likely degrade from extensive use of muscular effort. Perceptually, voices sounded "poorer" in quality, and mean airflow was reduced possibly because of phonotrauma and inappropriate use of perilaryngeal and respiratory muscles during performances. MSP time was substantially lower as well, although this was not statistically significant. Other measures did not differ to the point of statistical significance either, but changes in voice perception demonstrate clinical significance and potential degradation of voice in stage performances. Acoustic measures have not been clearly demonstrated to be effective measures of vocal function when compared with perceptual ratings. ${ }^{16}$ Thus, some of these measures may simply not be sensitive enough to detect what the perceptual raters were hearing. Regardless, our results are in agreement with the general premise of higher likelihood of phonotrauma in professional voice users. Specifically, the results complement findings reported in previous studies. ${ }^{6,7}$

The second hypothesis of potentially less impact in individuals with better practices of vocal hygiene and vocal training was, to some degree, unsubstantiated by the results. Higher alcohol consumption resulted in increased voice turbulence, perhaps because of dehydration effects. Likewise, inadequate water intake impacted phonation duration. Decreasing alcohol and increasing water are both part of vocal hygiene training - arguably, the two most common sense aspects of vocal hygiene for professional voice users. Previous research has yielded equivocal results about the use of vocal hygiene. Some reports ${ }^{17,18}$ suggest positive outcomes of vocal hygiene; however, others ${ }^{19}$ have reported no substantial benefit of vocal hygiene in professional voice users. Studies in voice actors in this regard are limited. Roy and colleagues ${ }^{12}$ reported some alleviation of vocal trauma by vocal hygiene; however, we did not observe similar trends.

Our results also suggest that vocal training does not have a substantial impact on phonotrauma in stage actors. This finding needs to be interpreted with caution because our questionnaire did not attempt to gather specific details about the training. In spite of this caveat, we did instruct participants to qualitatively summarize the kind of training they received and observed that participants who received any kind of training did not differ significantly in their vocal attributes from their counterparts who did not receive any training. These results are not in consonance with the limited studies reported previously. ${ }^{11}$ A larger sample of actors and more clearly defined questions regarding training could provide different results.

In consideration of the results in totality, it appears that stage actors are subjected to substantial phonotrauma similar to most elite vocal performers, as one would expect. This group of individuals is required to employ precise coordination of the speech subsystems coupled with excessive use to be able to perform on the stage. The constant use of muscles and increased effort, combined with environmental or psychological triggers, increase the likelihood of phonotrauma and subsequent development of voice disorders. Vocal hygiene knowledge, as reported by these actors, did not prove to be beneficial overall within the context of our design; however, some very basic, common sense aspects of vocal hygiene proved important: increasing water and decreasing alcohol consumption. Likewise, actors who basically live on the road with other actors likely receive peer professional voice training on a regular basis. Thus, many actors receive informal training that would not have been reported in our gathering of data. Prospective data specifically investigating the effects of a controlled vocal hygiene program in stage actors could be beneficial, as could more detailed data collection regarding knowledge of the principles espoused in professional voice training rather than questions regarding history of professional voice training.

\section{CONCLUSIONS}

Results of this investigation demonstrate that stage performances do impact vocal outcomes with reduction in quality and efficient use of airflow for voice production. Knowledge and practice of vocal hygiene have some impact on these changes; however, vocal hygiene may not be the best "shield" to prevent these changes. Research should continue to further identify and define best voice conservation methods in professional voice users, as well as specific aspects of informal and formal vocal training.

\section{Acknowledgments}

This work was funded by a University of Central Arkansas (UCA) Research Council Grant. Thanks to Hylan Pickett, MS, CCC-SLP for her help with perceptual assessment of voice samples. This study was partly supported by a grant from the National Institutes of Health/NIDCD R15 DC013137-01A1. 


\section{APPENDIX}

\section{Vocal Hygiene and Training Questionnaire}

\section{Profession}

1. Are you an: Actor Non-actor/Control

List Profession:

2. How would you describe your professional voice use?

I use it all the time, my profession depends on it.

I use it a lot but can get by without it much of the time.

It really only involves using my voice for some communication.

3. (Actors Only) How long have you been acting professionally?

Less than 1 year

$1-2$ years

3-4 years

$5-10$ years

more than 10 years

4. (Actors) How often do you act each year?

\section{Vocal Hygiene/Care of the Voice}

1. Have you received training on vocal hygiene/care of the voice? yes no

If yes, please describe when, where, and by whom.

2. What do you know about proper vocal hygiene, or care for the voice? Please list or describe anything you feel this involves.

3. How much water do you drink per day (8 ounces glasses)?

$$
\text { 1-2 glasses, _ 3-4 glasses, __ 5-6 glasses, __ more than } 6 \text { glasses }
$$

4. How much caffeine do you drink per day (equivalent to a cup of coffee)?

$$
\text { 1-2 cups, _ 3-4 cups, }
$$
5-6 cups, more than 6 cups

Please list these beverages:

5. Do you have allergies? _ yes _ no

If yes, do you take medication? Please list any allergy medications you take.

6. Do you have acid reflux? __ yes __ no

If yes, do you take medication? Please list any medications you take or things you do behaviorally to reduce reflux effects.

7. Do you smoke? _ yes __ no

If yes, please state what and how much.

8. How would you describe your normal speaking voice on average?

soft and easy

louder and more authoritative

regular and/or situation dependent

9. Please describe anything else you believe you do that may help or hurt your overall vocal health.

\section{Vocal Training (actors only)}

1. Have you ever received voice training/coaching?

yes no

If yes, for how long?

Please describe the training you received, when you received it, and where/by whom.

2. Do you use warm-up and/or cool-down voice exercises before and after performing?

If yes, please describe what you do?

3. Please list any other things you have been taught to do to help you maintain and/or maximize your voice.

\section{REFERENCES}

1. Shakespeare W. As You Like It. New Haven: Yale University Press; 1954.

2. Sapir S, Mathers-Schmidt B, Larson GW. Singers' and non-singers' vocal health, vocal behaviours, and attitudes towards voice and singing: indirect findings from a questionnaire. Int J Lang Commun Disord. 1996;31:193209.

3. Milutinović Z, Bojić P. Functional trauma of the vocal folds: classification and management strategies. Folia Phoniatr Logop. 1996;48:78-85.

4. Zeine L, Waltar KW. The voice and its care: survey findings from actors' perspectives. J Voice. 2002;16:229-243.
5. Eustace CS, Stemple JC, Lee L. Objective measures of voice production in patients complaining of laryngeal fatigue. J Voice. 1996;10:146-154.

6. Emerich KA, Titze IR, Švec JG, et al. Vocal range and intensity in actors: a studio versus stage comparison. J Voice. 2005;19:78-83.

7. Novak A, Dlouha O, Capkova B, et al. Voice fatigue after theater performance in actors. Folia Phoniatr Logop. 1991;43:74-78.

8. Kitch JA, Oates J. The perceptual features of vocal fatigue as self-reported by a group of actors and singers. $J$ Voice. 1994;8:207-214.

9. Behlau M, Oliveira G. Vocal hygiene for the voice professional. Curr Opin Otolaryngol Head Neck Surg. 2009;17:149-154. 
10. Nanjundeswaran C, Li NY, Chan KM, et al. Preliminary data on prevention and treatment of voice problems in student teachers. $J$ Voice. 2012;26:816, e1-12.

11. Laukkanen AM, Syrjä T, Laitala M, et al. Effects of two-month vocal exercising with and without spectral biofeedback on student actors' speaking voice. Logoped Phoniatr Vocol. 2004;29:66-76.

12. Roy N, Ryker KS, Bless DM. Vocal violence in actors: an investigation into its acoustic consequences and the effects of hygienic laryngeal release training. $J$ Voice. 2000;14:215-230.

13. Timmermans B, Bodt MD, Wuyts F, et al. Poor voice quality in future elite vocal performers and professional voice users. J Voice. 2002;16:372-382.

14. Kempster GB, Gerratt BR, Abbott KV, et al. Consensus auditory-perceptual evaluation of voice: development of a standardized clinical protocol. Am J Speech Lang Pathol. 2009;18:124-132.
15. Zraick RI, Smith-Olinde L, Shotts LL. Adult normative data for the KayPENTAX Phonatory Aerodynamic System Model 6600. J Voice. 2012;26:164-176.

16. Jotz GP, Cervantes O, Abrahão M, et al. Noise-to-harmonics ratio as an acoustic measure of voice disorders in boys. J Voice. 2002;16:28-31.

17. Chan RWK. Does the voice improve with vocal hygiene education? A study of some instrumental voice measures in a group of kindergarten teachers. J Voice. 1994;8:279-291.

18. Fletcher HM, Drinnan MJ, Carding PN. Voice care knowledge among clinicians and people with healthy voices or dysphonia. $J$ Voice. 2007;21:8091.

19. Broaddus-Lawrence PL, Treole K, McCabe RB, et al. The effects of preventive vocal hygiene education on the vocal hygiene habits and perceptual vocal characteristics of training singers. $J$ Voice. 2000;14:58-71. 